\title{
High rate of occult cancer found in prophylactic mastectomy specimens despite thorough presurgical assessment with MRI and ultrasound: findings from the Hereditary Breast and Ovarian Cancer Registration 2016 in Japan
}

\author{
Hideko Yamauchi ${ }^{1}$ (1) Megumi Okawa ${ }^{1}$. Shiro Yokoyama ${ }^{2} \cdot$ Chizuko Nakagawa $^{1} \cdot$ Reiko Yoshida $^{3} \cdot$ Koyu Suzuki $^{4}$. \\ Seigo Nakamura ${ }^{5} \cdot$ Masami Arai $^{6}$
}

Received: 28 August 2018 / Accepted: 1 September 2018 / Published online: 10 September 2018

(c) The Author(s) 2018

\begin{abstract}
Purpose Prophylactic surgery is a preemptive strategy for hereditary breast and ovarian cancer (HBOC). Prophylactic mastectomy (PM) reduces breast cancer risk by $>90 \%$. The aim of our study is to analyze the information of the Japanese pedigrees and to utilize the results for clinical practice.

Methods We statistically analyzed records of HBOC registrees who had undergone BRCA1/2 genetic testing at seven medical institutions up until 2016. In the cases of PM, we examined breasts with the use of mammography (MMG), ultrasound (US), and magnetic resonance imaging (MRI) before surgery. After PM, the specimens were divided about $1 \mathrm{~cm}$ serially and examined in their entirety.

Results Of 1527 registrees who underwent $B R C A$ testing, 1125 (73.7\%) were negative for BRCA1/2 mutation, 297 (19.5\%) were positive for $B R C A 1 / 2$ mutation $\left(B R C A 1 / 2^{M U T+}\right)$, and $105(6.9 \%)$ had uncertain results. To decide whether to undergo total mastectomy vs. breast-conserving surgery (BCS), 370 registrees underwent presurgical genetic testing. During the follow-up period, four new-onset breast cancers were found among the 55 non-affected BRCA carriers. Among the 73 $B R C A 1 / 2^{M U T+}$ carriers who underwent BCS, 3 were found to have ipsilateral breast cancer. Of $189 \mathrm{BRCAl/2^{MUT+ }}$ carriers with unilateral breast cancer, 8 were found to have contralateral breast cancer. Of 53 PM specimens, 6 (11.3\%) were found to have occult breast cancer despite using MMG, US, and MRI.

Conclusions Our report showed a relatively higher incidence rate of occult cancer at $11.3 \%$ in PM specimens despite thorough pre-operative radiological evaluations, which included a breast MRI. Considering the occult cancer rates and the various pathological methods of our study and published studies, we propose the necessity of a histopathological protocol.
\end{abstract}

Keywords BRCA · Hereditary breast and ovarian cancer syndrome - Magnetic resonance imaging - Occult cancer · Pathological method · Prophylactic mastectomy

\section{Introduction}

The breast cancer rate tends to peak at a younger range in Japan than in Western countries [1-3]. About half of breast cancer diagnoses in Japan are for patients in their 30s-50s [2]. As this pattern probably reflects their genetic background, investigations of hereditary breast and ovarian cancer (HBOC) are important for Japanese women. The

Hideko Yamauchi

hideyama@luke.ac.jp

Extended author information available on the last page of the article
Japanese nationwide HBOC registration system aims to clarify clinical and genetic features of Japanese HBOC and to improve its medical treatment.

The Japanese HBOC Consortium (JHC) was established in December 2012. We established a registration committee for JHC in October 2013 and promoted it as a nationwide registration project. The registered subjects were all Japanese individuals who underwent $B R C A l / 2$ genetic testing (including individuals in which no mutation was detected) [4]. Here, we report results of the HBOC Registration from its establishment until 2016. The objective of the current study is to analyze the information of the Japanese 
pedigrees, who underwent $B R C A 1 / 2$ genetic testing, and to make use of the results in clinical practice.

Prophylactic surgery, such as prophylactic mastectomy (PM), is a preemptive strategy for HBOC. As PM can reduce risk of breast cancer by $>90 \%$, it is often performed among BRCA1/2 mutation (BRCA1/2 ${ }^{M U T}$ ) carriers. Reportedly, occult cancers are detected in $0.5-9.9 \%$ of PM specimens [5-15].

\section{Methods}

This study included subjects who underwent $B R C A 1 / 2$ genetic testing until 2016. As of 2016, 7 participating medical institutions were enrolled: St. Luke's International Hospital (Tokyo), Cancer Institute Hospital (Tokyo), Showa University Hospital (Tokyo), Hoshi General Hospital (Fukushima), Kitano Hospital (Osaka), Shikoku Cancer Center (Ehime), and Kochi Medical School Hospital (Kochi).

All subjects, who received genetic counseling and underwent genetic testing of their own free will in clinical practice, were those who had been provided explanations of the HBOC risk in accordance with Genetic/Familial HighRisk Assessment: Breast and Ovarian in NCCN Guidelines [16]. Most of genetic testing with sequencing and large rearrangement analysis was performed at Myriad Genetic Laboratories or FALCO Biosystems. Detected variants were interpreted by the criteria of Myriad Genetic Laboratories. We entered information for BRCA1/2 genetic testing and clinicopathological findings of breast cancer, ovarian cancer, and other cancers in the original electronic template. All data except sex were anonymously registered in each institution. Dates of birth only included year and month [4].

In the cases of PM, we examined breasts with the use of mammography (MMG), ultrasound (US), and magnetic resonance imaging (MRI) before surgery. After PM, the specimens were processed by a pathologist. Although a surgicopathological protocol for occult cancer in the PM specimens does not exist, the specimens were divided about $1 \mathrm{~cm}$ serially and examined in their entirety.

\section{Results}

Of 1527 registrees who underwent $B R C A$ testing, 1125 (73.7\%) were negative for BRCA1/2 mutation (BRCA1/2 $2^{M U T-}$ ), 297 (19.5\%) were positive for BRCA1/2 mutation $\left(B R C A 1 / 2^{M U T+}\right)$, and $105(6.9 \%)$ had uncertain results. Among the $297 B R C A 1 / 2^{M U T+}$ subjects (19.5\%), 157 (10.3\%) carried mutations for BRCAl, 139 (9.1\%) for $B R C A 2$, and $1(0.1 \%)$ was positive for both (Fig. 1). Among 359 patients with triple-negative breast cancer, 101 (28.3\%) had mutations for BRCA1 and 18 (5.0\%) for BRCA2 (Fig. 2). Distribution of age at onset of breast cancer with/without BRCA1/2 mutations (Fig. 3) shows that BRCA1/2 ${ }^{M U T+}$ breast cancer occurred at a younger mean age (41.7 years) than did BRCA1/2 $2^{M U T-}$ breast cancer (45.8 years). In comparison to the 2013 National Registration for Breast Cancer Incidence

\section{Total 1527 cases}

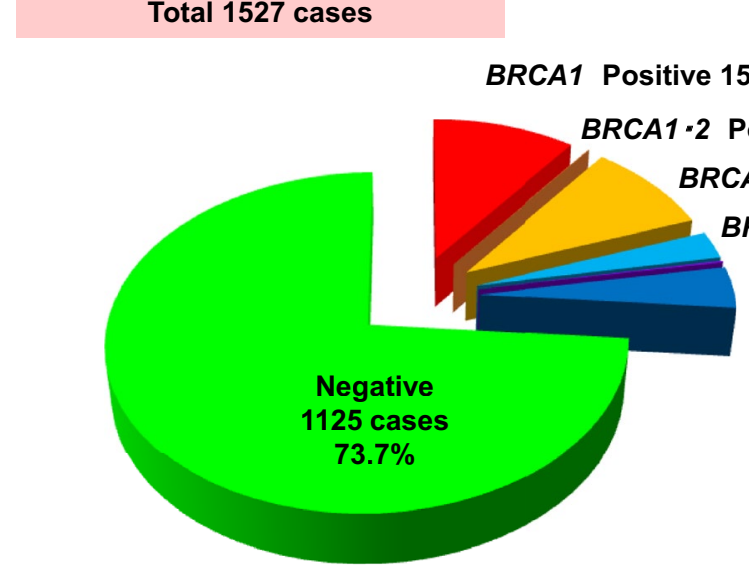

Mutation allele detection count

\begin{tabular}{|c|c|c|}
\hline & Positive & Uncertain \\
\hline BRCA1 & 158 & 48 \\
\hline BRCA2 & 140 & 72 \\
\hline
\end{tabular}

Fig. 1 Prevalence of $B R C A 1 / 2$ mutations 


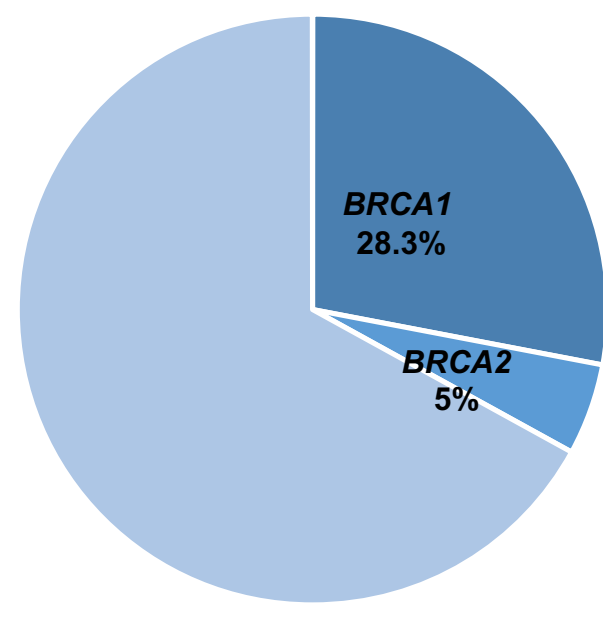

Fig. 2 Rates of $B R C A 1 / 2$ mutations in triple-negative breast cancers

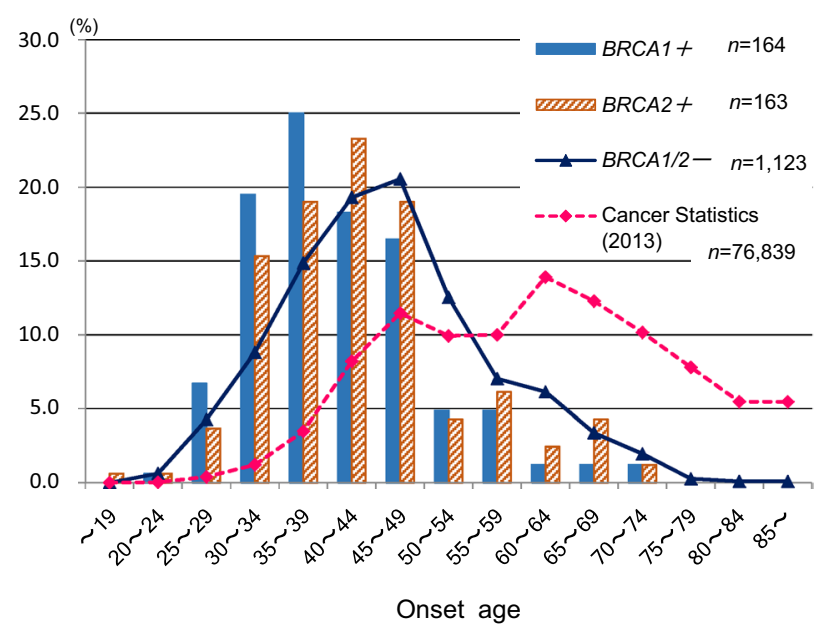

Fig. 3 Distribution of age at onset of breast cancer with/without BRCA1/2 mutations and national statistics (2013). Mean age at onset of breast cancer. BRCA1/2 mutation positive: 41.7 years; BRCA1/2 mutation negative: 45.8 years

in Japan $(n=76,839)$ [2], breast cancer with $B R C A$ mutations occurred at a younger age. Among types of $B R C A 1 / 2$ pathological mutations that were reported more than once, L63X was the most common (Table 1).

To help decide between surgical procedures (total mastectomy vs. breast-conserving surgery [BCS]), 370 subjects underwent presurgical genetic testing. Of the 66 BRCA1/2 $2^{M U T+}$ subjects, $58(87.9 \%)$ chose to undergo total mastectomy, and $8(12.1 \%)$ chose BCS. Of the 304 BRCA1/2 $2^{M U T-}$ subjects, $141(46.4 \%)$ chose total mastectomy, $158(52.0 \%)$ chose BCS, and 5 had unknown choices (Table 2).

During the follow-up period, four cases of newonset breast cancers were observed among the 55 nonaffected BRCA carriers (mean observation period: 2.5 years; incidence rate: $2.9 \%$ year; Table 3 ). Among the 73 $B R C A 1 / 2^{M U T+}$ women who underwent BCS, 3 ipsilateral breast cancer cases were found (mean observation period: 3.5 years; incidence rate: $1.2 \%$ year), compared with only 2 cases among the 477 BRCA1/2 $2^{M U T-}$ women (mean observation period: 2.2 years; incidence rate: $0.2 \%$ year; Table 4 ). Of $189 \mathrm{BRCA1/2^{MUT+ }}$ women with unilateral breast cancer, 8 contralateral breast cancer cases were found (mean observation period: 3.0 years; incidence rate: $1.4 \% /$ year), compared with 4 cases of contralateral breast cancer among 892 BRCA1/2 $2^{M U T-}$ women (mean observation period: 2.2 years; incidence rate: $0.2 \%$ year; Table 5).

Among the 51 patients who underwent PM (Table 6), 6 had specimens in which occult breast cancer was found, including 1 with a $B R C A 1$ mutation and 5 with $B R C A 2$ mutations. All six patients had undergone extensive imaging prior to PM, using MMG, US, and breast MRI (Tables 7, 8). In our study, the rate of occult cancer among total removed breasts by PM was $6 / 53=11.3 \%$.

\section{Discussions}

We report herein one of the highest incidence rates in the literature: $11.3 \%$ of occult cancer in PM specimens, despite thorough presurgical assessment with MRI, US, and MMG, compared with previously reported rates of $0.5-9.9 \%$ (Table 9). We reviewed several factors thought to influence occult cancer occurrence, including (a) rates of bilateral prophylactic mastectomy (BPM), (b) pre-PM examination methods, (c) BRCA1/2 $2^{M U T+}$ rates among subjects, and (d) pathological methods.

\section{Rates of BPM}

Rates of BPM among subjects in the first three studies of Table 9 are higher (at or near 100\%) than in the other studies. The retrospective study of Hartmann et al. [5] included all women with family histories of breast cancer who underwent BPM in USA between 1960 and 1993. They found only $0.5 \%$ of occult cancer after BPM, though the rate of $B R C A$ mutations among their subjects was not available. Meijers-Heijboer et al. [6] conducted a prospective study of 139 women with pathogenic $B R C A 1$ or $B R C A 2$ mutations who were enrolled in a breast-cancer surveillance program, Netherlands. Of the 139, 76 underwent PM from 1992 to 2001. They found only 1 case of lobular carcinoma in situ [LCIS (0.7\%)] and no cases of ductal carcinoma in situ (DCIS) or invasive ductal carcinoma (IDC), even among $B R C A 1 / 2^{M U T}$ carriers. The study of Yao et al. [7] was a retrospective review of pathology results and outcomes of 201 $B R C A 1 / 2^{M U T}$ carriers, in USA, treated between 2007 and 2014 (1.3\% occult cancer among 150 BRCA $1 / 2^{M U T}$ carriers 
Table 1 Types of $B R C A 1 / 2$ pathological mutations that were reported more than once

\begin{tabular}{|c|c|c|c|c|c|c|c|}
\hline \multicolumn{4}{|l|}{ BRCA1 } & \multicolumn{4}{|l|}{$B R C A 2$} \\
\hline \multicolumn{2}{|l|}{ Base mutation } & \multirow[t]{2}{*}{ Amino acid mutation } & \multirow[t]{2}{*}{ Report count } & \multicolumn{2}{|l|}{ Base mutation } & \multirow[t]{2}{*}{ Amino acid mutation } & \multirow[t]{2}{*}{ Report count } \\
\hline Myriad & HGVS & & & Myriad & HGVS & & \\
\hline $307 \mathrm{~T}>\mathrm{A}$ & c. $188 \mathrm{~T}>\mathrm{A}$ & L63X & 40 & $5804 \mathrm{del} 4$ & c.5576_5579delTTAA & STOP1862 & 10 \\
\hline Unconfirmed & & & 1 & $7180 \mathrm{C}>\mathrm{T}$ & c. $6952 \mathrm{C}>\mathrm{T}$ & $\mathrm{R} 2318 \mathrm{X}$ & 9 \\
\hline $2919 \mathrm{C}>\mathrm{T}$ & c. $2800 \mathrm{C}>\mathrm{T}$ & Q934X & 13 & $8732 \mathrm{C}>\mathrm{A}$ & c. $8504 \mathrm{C}>\mathrm{A}$ & S2835X & 7 \\
\hline 3561delG & c.3442delG & STOP1154 & 5 & Unconfirmed & & & 1 \\
\hline 575delCA & c.456_457delCA & STOP157 & 5 & $9345 \mathrm{G}>\mathrm{A}$ & c. $9117 \mathrm{G}>\mathrm{A}$ & P3039P & 6 \\
\hline 2508delGA & c.2389_2390delGA & STOP799 & 4 & 1506delA & c.1278delA & STOP429 & 6 \\
\hline $3759 \mathrm{G}>\mathrm{T}$ & c. $3640 \mathrm{G}>\mathrm{T}$ & E1214X & 4 & $8857 \mathrm{G}>\mathrm{T}$ & c. $8629 \mathrm{G}>\mathrm{T}$ & E2877X & 5 \\
\hline $5083 \mathrm{C}>\mathrm{T}^{\mathrm{b}}$ & c. $4964 \mathrm{C}>\mathrm{T}$ & $\mathrm{S} 1655 \mathrm{~F}$ & 4 & $9304 \mathrm{C}>\mathrm{T}$ & c. $9076 \mathrm{C}>\mathrm{T}$ & Q3026X & 5 \\
\hline IVS20-1G $>A$ & c. $5278-1 \mathrm{G}>\mathrm{A}$ & & 3 & $5873 \mathrm{C}>\mathrm{A}$ & c. $5645 \mathrm{C}>\mathrm{A}$ & S1882X & 5 \\
\hline IVS20-1G $>C$ & c. $5278-1 \mathrm{G}>\mathrm{C}$ & & 3 & 8817insA & c.8589dupA & STOP2868 & 5 \\
\hline $1623 \mathrm{del} 5$ & c.1504_1508delTTAAA & STOP503 & 2 & 2041delA & c.1813dupA & STOP613 & 5 \\
\hline $297 \mathrm{C}>\mathrm{T}$ & c. $178 \mathrm{C}>\mathrm{T}$ & Q60X & 2 & 5804del4 & c.5576_5579delTTAA & STOP1861 & 4 \\
\hline $309 \mathrm{~T}>\mathrm{C}$ & c. $190 \mathrm{~T}>\mathrm{C}$ & C64R & 2 & $9610 \mathrm{C}>\mathrm{T}$ & c. $9382 \mathrm{C}>\mathrm{T}$ & $\mathrm{R} 3128 \mathrm{X}$ & 3 \\
\hline $5181 \mathrm{del} 3$ & c.5062delGTT & V1688del & 2 & 3463delT & c.3235delT & STOP1086 & 3 \\
\hline $5280 \mathrm{C}>\mathrm{T}$ & c. $5161 \mathrm{C}>\mathrm{T}$ & Q1721X & 2 & 983del4 & c.755_758delACAG & STOP275 & 3 \\
\hline exon1a-2del & $\begin{array}{l}\text { c.(?_-1387-1)__ } \\
\quad\left(80+1 \_81-1\right) \text { del }\end{array}$ & & 2 & $4123 \mathrm{G}>\mathrm{T}$ & c. $3895 \mathrm{G}>\mathrm{T}$ & E1299X & 2 \\
\hline exon8 del & $\begin{array}{l}\text { c. }\left(441+11_{1} 442-1\right)_{-} \\
\quad\left(546+1 \_547-1\right) \text { del }\end{array}$ & & 2 & $8251 \mathrm{~A}>\mathrm{G}^{\mathrm{b}}$ & c. $8023 \mathrm{~A}>\mathrm{G}$ & $\mathrm{I} 2675 \mathrm{~V}$ & 2 \\
\hline \multirow[t]{3}{*}{ IVS14-2A $>G^{a}$} & c. $4485-2 A>G$ & & 2 & 3423del4 & c.3195_3198delTAAT & STOP1075 & 2 \\
\hline & & & & 2041 ins A & c.1813dupA & STOP615 & 2 \\
\hline & & & & 3036del4 & c. $2808 \_2811$ delACAA & STOP959 & 2 \\
\hline
\end{tabular}

${ }^{\text {a }}$ Suspected deleterious

${ }^{\mathrm{b}}$ Mixed suspected deleterious and deleterious

Table 2 Genetic testing to select breast cancer surgical procedures $(n=418)$

\begin{tabular}{lllll}
\hline Testing results & Cases count & \multicolumn{2}{l}{ Breast cancer operation type } \\
\cline { 3 - 5 } & & $\begin{array}{l}\text { Breast- } \\
\text { conserving } \\
\text { surgery }\end{array}$ & Mastectomy & Unknown \\
& & 8 & 58 & 0 \\
\hline Positive & 66 & $12.1 \%$ & $87.9 \%$ & $0 \%$ \\
Negative & 304 & 158 & 141 & 5 \\
& & $52.0 \%$ & $46.4 \%$ & $1.6 \%$ \\
\hline
\end{tabular}

\# of 418 patients, 370 underwent surgery after genetic testing

[298 breasts] undergoing nipple-sparing PMs). The much higher rates of BPM in these three studies seem to have resulted in much lower occult cancer rates $(0.5-1.3 \%)$ than in the other studies. BPM patients are considered to have no history of breast cancer.

In contrast, van Sprundel et al. [10] in the Netherlands found 5\% occult cancer among 79 of 148 patients who underwent contralateral prophylactic mastectomy (CPM). The 148 patients were identified until June 2003 as carrying $B R C A 1$ or $B R C A 2$ mutations with previous histories of unilateral, stage I-IIIa invasive breast cancer. Evans et al. [12] in UK between 1985 and 2010, considered whether CPM improves overall survival, and found $5.7 \%$ occult cancer in 105 women with $B R C A 1 / 2$ mutations and unilateral breast cancer who underwent CPM. By comparing the $100 \%$ (or near-100\%) BPM cohorts with 100\% CPM cohorts, we see that high rates of BPM might be associated with lower rates of occult cancer. Therefore, even among BRCA carriers, detection rate of occult cancer may have been influenced by the status whether affected or non-affected.

\section{Pre-PM examination methods}

Black et al. [15] of USA reviewed occult malignancy in 192 PMs in 173 patients treated from 1999 to 2005, to compare pre-operative MRI with sentinel lymph node biopsy (SLNB), and found that MRI (performed in 59 patients) missed three of four total occult cancers. In the study of McLaughlin et al. [11] (USA) of 529 patients who underwent 613 PMs between 1999 and 2006, both pre-operative MRI and SLNB were performed selectively at the discretion of the surgeon; they reported the sensitivity of MRI for detecting occult cancers to be $78 \%$. In a 2015 study, Riedl et al. [17] insisted that the use of MRI to screen women at increased risk for breast cancer improved 
Table 3 Breast cancer after genetic testing among nonaffected $B R C A$ carriers
Table 4 Breast cancers in ipsilateral breasts after breastconserving surgery
Table 5 Contralateral breast cancers among patients treated for unilateral breast cancers

\begin{tabular}{ll}
\hline Carriers without a history of breast cancer & \\
\hline Observation period after genetic testing (average) & 55 cases \\
Age at genetic testing (average) & $0-13.9$ years (2.5) \\
Breast cancer onset after genetic testing & Age 20-66 (age 38.6) \\
Incidence rate & 4 cases \\
& $4 / 137.5$ (persons/person years) \\
BRCA1 positive 2 cases, $\boldsymbol{B R C A 2}$ positive 2 cases & $2.9 \% /$ year \\
Opportunities for detection & \\
& MRI: 2 cases, DCIS \\
& MMG: 1 case, DCIS \\
& Self-detection: 1 case, invasive $3.2 \mathrm{~cm}$
\end{tabular}

$D C I S$ ductal carcinoma in situ, $M M G$ mammography, $M R I$ magnetic resonance imaging

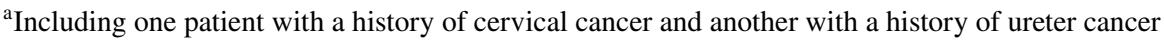

\begin{tabular}{lll}
\hline & BRCA1/2 positive & BRCA1/2 negative \\
\hline Women with a history of breast-conserving surgery & 73 cases & 477 cases \\
Ipsilateral breast cancer onset after genetic testing & 3 cases & 2 cases \\
Observation period after genetic testing (average) & $0.01-12.3$ years (3.5) & $0.01-12.5$ years (2.2) \\
Incidence rate & $3 / 256$ & $2 / 1049$ \\
& (persons/person years) & (persons/person years) \\
& $1.2 \% /$ year & $0.2 \% /$ year \\
Background & & Age 19-71 (age 41.7) \\
Age of onset of the first breast cancer (average) & Age 46.4) \\
Number of exclusion cases due to PM & 3 cases & 0 cases \\
\hline
\end{tabular}

$P M$ prophylactic mastectomy

\begin{tabular}{lll}
\hline & BRCA1/2 positive & BRCA1/2 negative \\
\hline Women with a history of unilateral breast cancer & 189 cases & 892 cases \\
Contralateral breast cancer onset after genetic testing & 8 cases & 4 cases \\
Observation period after genetic testing (average) & $0.02-16.8$ years & $0.01-20.2$ years \\
& $(3.0)$ & $(2.2)$ \\
Incidence rate & $8 / 567$ & $4 / 1962$ \\
& (persons/person years) & (persons/person years) \\
& $1.4 \% /$ year & $0.2 \% /$ year \\
Background & & Age 19-74 (age 41.7) \\
Age of onset of the first breast cancer (average) & Age (age 45.4) \\
Number of exclusion cases due to PM & 37 cases & 3 cases \\
\hline
\end{tabular}

$P M$ prophylactic mastectomy detection of invasive cancers and DCIS, regardless of mutation status, age, or breast density; their improved results for MRI sensitivity might be explained by technical advances, improved diagnostic criteria, and greater familiarity of radiologists in reading breast MRIs, including the ability to diagnose DCIS with MRI [17, 18].

Regarding US, Bosse et al. [19] reported with respect to $B R C A 1 / 2^{M U T}$ carriers, that the sensitivity of US was $77 \%$, and that of MRI was $100 \%$. Ohuchi et al. [20] from Japan reported that the sensitivity of MMG + US for asymptomatic women aged 40-49 years with no history of any cancer in the previous 5 years was $91.1 \%$.

With regard to MRI + MMG + US (yearly MRI, MMG, and biannual US), van Zelst et al. [21] reported the sensitivity to be $76.3 \%$ for surveillance of BRCA1/2 ${ }^{M u t+}$ women and their first-degree untested relatives. Riedl et al. [17] reported the sensitivity to be $95.0 \%$, among BRCA1/2 $2^{M u t+}$ carriers and women with a familial risk $>20 \%$ (US was offered to BRCA mutation carriers). Kuhl et al. [22] reported the sensitivity to be $100 \%$, in a high-risk population (370 of 687 patients underwent US). Our study 
Table 6 Clinicopathological characteristics of patients who underwent prophylactic mastectomies $(n=51)$

\begin{tabular}{ll}
\hline Age & \\
Mean & 43.7 \\
Median & 43 \\
Range & $30-62$ \\
BRCA1 & \\
Positive & 29 cases \\
BRCA2 & \\
Positive & 18 cases \\
BRCA1/2 & \\
Negative & 4 cases \\
Breast cancer stage & \\
0 & 3 cases \\
1 & 17 cases \\
2 & 14 cases \\
3 & 4 cases \\
4 & 0 case \\
Non-onset & 2 cases \\
Uncertain & 11 cases \\
Breast cancer subtype & \\
Hormone positive & \\
HER2 negative & 11 cases \\
HER2 positive & 3 cases \\
HER2 uncertain & 8 cases \\
Hormone negative & \\
HER2 positive & 0 case \\
HER2 negative & 23 cases \\
HER2 uncertain & 2 cases \\
Non-onset & 2 cases \\
Uncertain & 2 cases \\
\hline & \\
$H E R 2 ~ h u m n$
\end{tabular}

HER2 human epidermal growth factor receptor 2

\begin{tabular}{ll}
\hline Age & \\
Mean & 42.2 \\
Median & 43 \\
Range & $33-51$ \\
BRCA1 & 1 case \\
BRCA2 & 5 cases \\
Occult cancer & \\
DCIS & 5 cases \\
Invasive cancer & 1 case \\
\hline DCIS ductal carcin
\end{tabular}

DCIS ductal carcinoma in situ

Table 7 Clinicopathological characteristics of patients in whom occult cancer was found after undergoing prohylactic mastectomies
Table 8 Cases of occult cancer in this study

\begin{tabular}{lllll}
\hline & Age & BRCA1 or 2 & Size $(\mathrm{cm})$ & Type \\
\hline 1 & 36 & 2 & NA & DCIS \\
2 & 47 & 2 & NA & DCIS \\
3 & 43 & 1 & NA & DCIS \\
4 & 51 & 2 & 0.5 & Invasive \\
5 & 43 & 2 & NA & DCIS \\
6 & 33 & 2 & NA & DCIS \\
\hline
\end{tabular}

DCIS ductal carcinoma in situ, NA not available

\section{BRCA $1 / 2^{\mathrm{MUT}+}$ rates}

BRCA mutation rates and occult cancer rates do not seem to be related in the studies cited in Table 9. For example, in the study of Burger et al. [8] on women who underwent PM ( $n=83$ in 71 patients) and SLNB ( $n=1522$ in 1498 patients) between 2005 and 2010 in UK, the rate of BRCA mutation in the 71 patients was $8.5 \%$ and the occult cancer rate was $4.8 \%$, which is similar to $5 \%$ reported by van Sprundel et al. [10] among a $100 \%$ BRCA1/2 $2^{M U T+}$ population. Kauff et al. [14] compared prevalence of histopathologic lesions in PM (performed between 1987 and 2001 in USA) specimens from women with $B R C A$ mutations and in age and race-matched cadaver mastectomy specimens and found that high-risk epithelial proliferative lesions (including DCIS) are more common in the unaffected breasts of women with known $B R C A$ mutations than in women of the comparison group. However, they said that determining whether these lesions are more common in women with $B R C A$ mutations than in those without will require direct comparison to women without mutations or with low risk for carrying mutations.

\section{Pathological methods}

Pathological examination methods vary among the papers cited in Table 9. Some studies evaluated 2-4 sections per quadrant of the breast and a section of the nipple, and another evaluated them by $5 \mathrm{~mm}$ slices and radiological examination; the methods of the others are not known. Boughey et al. [9] examined specimens (from PMs, between 2000 and 2005, USA) of at least 2 sections per each quadrant and nipple; the specimens were also macroscopically sliced and any areas found abnormal by palpation were evaluated further at the pathologists' discretion. They noted a 5\% occult cancer rate, including 2 IDCs. In the study of Hoogerbrugge et al. [13], the specimens (from PM between 1989 and 2001, Netherlands) were cooled and sliced in serial sections with approximately $5 \mathrm{~mm}$ intervals. Radiographs were made from the tissue slices. Suspicious lesions and randomly selected areas from each quadrant and the nipple were sampled, with a mean number of 19 samples per specimen. With this method, they detected 
Table 9 Occult cancers reported in the literature

\begin{tabular}{|c|c|c|c|c|c|c|c|}
\hline References & Subjects\# & $\%$ of BRCA & \# of BPM & \# of Total PM & $\begin{array}{l}\text { Occult cancer rate by } \\
\text { total PM\# }\end{array}$ & Pre-PM exam & Pathological method \\
\hline Hartmann [5] & 645 & NA & 645 & 1290 & $6 / 1290(0.5 \%)$ & NA & NA \\
\hline Meijers-Heijboer [6] & 76 & 100 & 76 & 152 & $\begin{array}{l}1 / 152(0.7 \%) \\
\text { LCIS: } 1, \text { No DCIS } \\
\text { or IDC }\end{array}$ & PE, MMG, or MRI & $\begin{array}{l}3 \text { random blocks/ } \\
\text { quadrant }\end{array}$ \\
\hline Yao [7] & 150 & 100 & 148 & 298 & $\begin{array}{l}4 / 298(1.3 \%) \\
\text { IDC:1, DCIS:3 }\end{array}$ & $\begin{array}{l}\text { PE, MMG, or US, } \\
\text { All MRI }\end{array}$ & NA \\
\hline Burger [8] & 71 & 8.5 & 12 & 83 & $\begin{array}{l}4 / 83(4.8 \%) \\
\text { ILC }(3.5 \mathrm{~mm}): 1, \\
\text { LCIS:3 }\end{array}$ & NA & NA \\
\hline Boughey [9] & 409 & 5.6 & 27 & 436 & $\begin{array}{l}22 / 436(5.0 \%) \\
\text { IDC:2, ILC:6 } \\
\text { (IDC\&ILC:2-9 mm) } \\
\text { DCIS: } 14\end{array}$ & PE, MMG & $\begin{array}{l}2 \text { section/each quad- } \\
\text { rant \& nipple }\end{array}$ \\
\hline van Sprundel [10] & 79 & 100 & 0 & 79 & $\begin{array}{l}4 / 79(5.1 \%) \\
\text { IDC(32 mm):1, } \\
\text { DCIS:3 }\end{array}$ & $\mathrm{PE}$, radiological & NA \\
\hline McLaughlin [11] & 529 & 9.3 & 84 & 613 & $\begin{array}{l}\text { 33/613 (5.4\%) } \\
\text { IDC:10, DCIS:23 }\end{array}$ & $\begin{array}{l}\text { PE, MMG, (US } \\
\text { and/or MRI), } \\
\text { (235/529pts: MRI) }\end{array}$ & $\begin{array}{l}2 \text { section/each quad- } \\
\text { rant \& nipple }\end{array}$ \\
\hline Evans [12] & 105 & 100 & 0 & 105 & $\begin{array}{l}\text { 6/105 (5.7\%) } \\
\text { IDC:4, DCIS:2 }\end{array}$ & NA & NA \\
\hline Hoogerbrugge [13] & 67 & 66 & 41 & 108 & $\begin{array}{l}\text { 10/108 }(9.3 \%) \\
\text { IDC }(4 \mathrm{~mm}): 1 \\
\text { DCIS }(2-40 \mathrm{~mm}): 9 \\
(17 / 67 \text { pts: LCIS })^{\mathrm{a}}\end{array}$ & $\begin{array}{l}\text { PE, MMG, 4/10pts } \\
\text { MRI, (27/67pts: } \\
\text { MRI) }\end{array}$ & $\begin{array}{l}5 \mathrm{~mm} \text { slices and } \\
\text { radiological exam, } \\
\text { then suspicious } \\
\text { lesions and ran- } \\
\text { domly selected each } \\
\text { quadrant and nipple } \\
\text { (Ave. } 19 \text { slides) }\end{array}$ \\
\hline Kauff [14] & 24 & 100 & 7 & 31 & $\begin{array}{l}3 / 31(9.7 \%) \\
\text { DCIS }(7-20 \mathrm{~mm}): 3 \\
(\text { LCIS: } 1)^{\mathrm{a}}\end{array}$ & MMG & $\begin{array}{l}\text { 2-4 section/each } \\
\text { quadrant\& nipple }\end{array}$ \\
\hline Black [15] & 173 & 17 & 19 & 192 & $\begin{array}{l}\text { 19/192 }(9.9 \%) \\
\text { IDC }(1.5-10 \mathrm{~mm}): 5 \text {, } \\
\text { DCIS: } 14\end{array}$ & 59/173pts MRI & NA \\
\hline Our study & 51 & 92 & 2 & 53 & $\begin{array}{l}6 / 53(11.3 \%) \\
\text { IDC }(5 \mathrm{~mm}): 1, \\
\text { DCIS:5 }\end{array}$ & $\begin{array}{l}\text { PE, MMG, US \& } \\
\text { MRI }\end{array}$ & About $1 \mathrm{~cm}$ slices \\
\hline
\end{tabular}

$B P M$ bilateral prophylactic mastectomy, DCIS ductal carcinoma in situ, IDC invasive ductal carcinoma, $I L C$ invasive lobular carcinoma, $L C I S$ lobular carcinoma in situ, $M M G$ mammography, $M R I$ magnetic resonance imaging, $N A$ not available, $P E$ physical examination, $P M$ prophylactic mastectomy, US ultrasound

${ }^{a}$ LCIS were detected, but not included, as occult cancer cases, as they may co-exist with DCIS

9.3\% occult cancer including one $4 \mathrm{~mm}$ IDC, and the occult cancer rate would have been higher than $9.3 \%$ if LCIS were counted. In the other studies, more detailed pathological examinations might have detected higher occult cancer rates. In the current study, we divided PM specimens about $1 \mathrm{~cm}$ serially and then, examined their entirety, and noted $11.3 \%$ occult cancer, including a $5 \mathrm{~mm}$ IDC. At present, there is not a strict histopathological protocol for PM specimens. However, there exist pathological guidelines for ovarian cancer [23]. Similarly, standardized guidelines for examining PM specimens may be required, as we might easily have missed the aforementioned5-mm occult cancer. In addition to this, occult cancer should be defined in the protocol, for example, as to whether LCIS can be included in the occult cancer.

This investigation is limited by the fact that it is a registration study from seven institutions and may not fairly reflect the entire population of Japanese BRCA mutation carriers.

\section{Conclusions}

Our report showed a relatively higher incidence rate of occult cancer at $11.3 \%$ in PM specimens despite thorough pre-operative radiological evaluations, which included a 
breast MRI. Considering the occult cancer rates and the various pathological methods of our study and published studies, we propose the necessity of a histopathological protocol.

Acknowledgements We are grateful to Dr. Akihiro Sakurai (Sapporo Medical University), Drs. Takayuki Enomoto and Masayuki Sekine (Niigata University Graduate School of Medical and Dental Sciences), Dr. Tadashi Nomizu (Hoshi General Hospital), and Dr. Junko Yotsumoto (Ochanomizu University) for supporting us as members of the registration committee of the Japanese HBOC Consortium.

Funding This work was supported by Health, Labour and Welfare Sciences Research Grants (H29-policy for cancer-general-003) and AMED (18 cm0106503h0003).

\section{Compliance with ethical standards}

Conflict of interest The authors claim no conflicts of interest.

Ethical approval All procedures performed in studies involving human participants were in accordance with the ethical standards of the Japanese HBOC Consortium Research Committee and with the 1964 Helsinki Declaration and its later amendments or comparable ethical standards.

Informed consent Informed consent was obtained from all individual participants included in the study.

Open Access This article is distributed under the terms of the Creative Commons Attribution 4.0 International License (http://creativeco mmons.org/licenses/by/4.0/), which permits unrestricted use, distribution, and reproduction in any medium, provided you give appropriate credit to the original author(s) and the source, provide a link to the Creative Commons license, and indicate if changes were made.

\section{References}

1. Saika K, Sobue T (2009) Epidemiology of breast cancer in Japan and the US. Jpn Med Assoc J (JMAJ) 52(1):39-44. https://www. med.or.jp/english/journal/pdf/2009_01/039_044.pdf

2. Center for Cancer Control and Information Services. Graph Database [Cancer Information Services For Health Professionals]. http://gdb.ganjoho.jp Accessed 2 May 2018

3. Leong SPL, Shen ZZ, Liu TJ et al (2010) Is breast cancer the same disease in Asian and Western countries? World J Surg 34(10):2308-2324. https://doi.org/10.1007/s00268-010-0683-1

4. Arai M, Yokoyama S, Watanabe C et al (2018) Genetic and clinical characteristics in Japanese hereditary breast and ovarian cancer: first report after establishment of HBOC registration system in Japan. J Hum Genet 63(4):447-457. https://doi.org/10.1038/ s10038-017-0355-1

5. Hartmann LC, Schaid DJ, Woods JE et al (1999) Efficacy of bilateral prophylactic mastectomy in women with a family history of breast cancer. N Engl J Med 340(2):77-84

6. Meijers-Heijboer H, van Geel B, van Putten WLJ et al (2001) Breast cancer after prophylactic bilateral mastectomy in women with a BRCA1 or BRCA2 mutation. N Engl J Med 345(3):159164. https://doi.org/10.1056/NEJM200107193450301

7. Yao K, Liederbach E, Tang R et al (2014) Nipple-sparing mastectomy in BRCA1/2 mutation carriers: an interim analysis and review of the literature. Ann Surg Oncol 22(2):370-376. https:// doi.org/10.1245/s10434-014-3883-3

8. Burger A, Thurtle D, Owen S et al (2013) Sentinel lymph node biopsy for risk-reducing mastectomy. Breast J 19(5):529-532. https://doi.org/10.1111/tbj.12157

9. Boughey JC, Khakpour N, Meric-Bernstam F et al (2006) Selective use of sentinel lymph node surgery during prophylactic mastectomy. Cancer 107(7):1440-1447. https://doi.org/10.1002/ cncr.22176

10. van Sprundel TC, Schmidt MK, Rookus MA et al (2005) Risk reduction of contralateral breast cancer and survival after contralateral prophylactic mastectomy in BRCA1 or BRCA2 mutation carriers. Br J Cancer 93(3):287-292. https://doi.org/10.1038/ sj.bjc.6602703

11. McLaughlin SA, Stempel M, Morris EA, Liberman L, King TA (2008) Can magnetic resonance imaging be used to select patients for sentinel lymph node biopsy in prophylactic mastectomy? Cancer 112(6):1214-1221. https://doi.org/10.1002/cncr.23298

12. Evans DG, Ingham SL, Baildam A et al (2013) Contralateral mastectomy improves survival in women with $B R C A 1 / 2$-associated breast cancer. Breast Cancer Res Treat 140(1):135-142. https:// doi.org/10.1007/s10549-013-2583-1

13. Hoogerbrugge N, Bult $\mathrm{P}$, de Widt-Levert LM et al (2003) High prevalence of premalignant lesions in prophylactically removed breasts from women at hereditary risk for breast cancer. J Clin Oncol 21(1):41-45. https://doi.org/10.1200/JCO.2003.02.137

14. Kauff ND, Brogi E, Scheuer L et al (2003) Epithelial lesions in prophylactic mastectomy specimens from women with $B R C A$ mutations. Cancer 97(7):1601-1608. https://doi.org/10.1002/ cncr. 11225

15. Black D, Specht M, Lee JM et al (2007) Detecting occult malignancy in prophylactic mastectomy: preoperative MRI versus sentinel lymph node biopsy. Ann Surg Oncol 14(9):2477-2484. https ://doi.org/10.1245/s10434-007-9356-1

16. National Comprehensive Cancer Network. NCCN guidelines for detection, prevention, \& risk reduction. https://www.ncen.org/

17. Riedl CC, Luft N, Bernhart C et al (2015) Triple-modality screening trial for familial breast cancer underlines the importance of magnetic resonance imaging and questions the role of mammography and ultrasound regardless of patient mutation status, age, and breast density. J Clin Oncol 33(10):1128-1135. https://doi. org/10.1200/JCO.2014.56.8626

18. Warner E, Causer PA, Wong JW et al (2011)) Improvement in DCIS detection rates by MRI over time in a high-risk breast screening study. Breast J 17(1):9-17. https://doi.org/10.111 1/j.1524-4741.2010.01018.x

19. Bosse K, Graeser M, Goßmann A, Hackenbroch M, Schmutzler RK, Rhiem K (2014) Supplemental screening ultrasound increases cancer detection yield in BRCA1 and BRCA2 mutation carriers. Arch Gynecol Obstet 289(3):663-670. https://doi.org/10.1007/ s00404-013-3022-6

20. Ohuchi N, Suzuki A, Sobue T et al, J-START investigator groups (2016) Sensitivity and specificity of mammography and adjunctive ultrasonography to screen for breast cancer in the Japan Strategic Anti-cancer Randomized Trial (J-START): a randomised controlled trial. Lancet 387(10016):341-348. https:// doi.org/10.1016/S0140-6736(15)00774-6

21. van Zelst JCM, Mus RDM, Woldringh G et al (2017)) Surveillance of women with the BRCA1 or BRCA2 mutation by using biannual automated breast US, MR imaging, and mammography. Radiology 285(2):376-388. https://doi.org/10.1148/radiol.20171 61218

22. Kuhl C, Weigel S, Schrading S et al (2010) Prospective multicenter cohort study to refine management recommendations for women at elevated familial risk of breast cancer: the EVA 
trial. J Clin Oncol 28(9):1450-1457. https://doi.org/10.1200/ JCO.2009.23.0839

23. Rabban JT, Krasik E, Chen LM, Powell CB, Crawford B, Zaloudek CJ (2009) Multistep level sections to detect occult fallopian tube carcinoma in risk-reducing salpingo-oophorectomies from women with BRCA mutations: implications for defining an optimal specimen dissection protocol. Am J Surg Pathol 33:1878-1885. https ://doi.org/10.1097/PAS.0b013e3181bc6059

\section{Affiliations}

\section{Hideko Yamauchi ${ }^{1}$ (D) Megumi Okawa ${ }^{1} \cdot$ Shiro Yokoyama $^{2} \cdot$ Chizuko Nakagawa $^{1} \cdot$ Reiko Yoshida $^{3} \cdot$ Koyu Suzuki $^{4}$. Seigo Nakamura ${ }^{5} \cdot$ Masami Arai $^{6}$}

1 Department of Breast Surgical Oncology, St. Luke's International Hospital, 9-1 Akashi-cho, Chuo-ku, Tokyo 104-8560, Japan

2 The Japanese HBOC Consortium, Division of Breast Surgical Oncology, Department of Surgery, Showa University School of Medicine, 1-5-8 Hatanodai, Shinagawa-ku, Tokyo 142-8666, Japan

3 Clinical Genetic Oncology, Cancer Institute Hospital of Japanese Foundation for Cancer Research, 3-8-31, Ariake, Koto-ku, Tokyo 135-8550, Japan
4 Department of Pathology, St. Luke's International Hospital, 9-1 Akashi-cho, Chuo-ku, Tokyo 104-8560, Japan

5 Division of Breast Surgical Oncology, Department of Surgery, Showa University School of Medicine, 1-5-8 Hatanodai, Shinagawa-ku, Tokyo 142-8666, Japan

6 Diagnostics and Therapeutics of Intractable Disease, Juntendo University, Graduate School of Medicine, 2-1-1 Hongo, Bunkyo-ku, Tokyo 113-8421, Japan 